The University of San Francisco

USF Scholarship: a digital repository @ Gleeson Library | Geschke Center

Nursing and Health Professions Faculty Research and Publications

School of Nursing and Health Professions

$3-2012$

\title{
Overview of Smart Phone Video Essentials
}

Scott Erdley

Margaret M. Hansen EdD, MSN, RN

University of San Francisco, mhansen@usfca.edu

Follow this and additional works at: http://repository.usfca.edu/nursing_fac

Part of the Nursing Commons

\section{Recommended Citation}

Erdley, Scott and Hansen, Margaret M. EdD, MSN, RN, "Overview of Smart Phone Video Essentials" (2012). Nursing and Health Professions Faculty Research and Publications. Paper 15.

http://repository.usfca.edu/nursing_fac/15 Gleeson Library | Geschke Center. It has been accepted for inclusion in Nursing and Health Professions Faculty Research and Publications by an authorized administrator of USF Scholarship: a digital repository @ Gleeson Library | Geschke Center. For more information, please contact repository@usfca.edu. 


\section{Overview of Smart Phone Video Essentials}

- Apple's iPhone

- Android-powered Smartphone

- Flip Video

This paper provides a practical review of the more common available smartphones along with a popular handheld video recorder. Their respective abilities to produce credible video recordings for use in formal nursing education as well as staff development are described. Reviewed are two of the more common smartphone operating systems, Apple's iPhone and the Android-powered smartphone. There were included because they are the most popular smartphone operating systems in the U.S. with video recording and editing capabilities. The smartphone operating systems highlighted in this review, are becoming more and more the device of choice for many healthcare professionals. Because of its popularity, as well as its userfriendly operation, the Flip Video was also included.

Film production includes planning, recording content, and editing. In the past, this has been time intensive because production required recording devices such as microphones, digital cameras, and software to edit audio and video segments. The devices reviewed for this paper compress all of these technologies into a small footprint and are low-cost and easy-to-use mobile tools. The authors' experience with each item, basic product technical information, and actual, as well as potential uses will be included. There is an easy to read table at the end of this article comparing each device on several aspects. The Flip Video will be reviewed first followed by the Apple iPhone and then the Android smartphone.

\section{Flip Video ${ }^{\text {Tw }}$}


The Flip Video ${ }^{\mathrm{TM}}$ is a pocketsize film-capturing device now manufactured by Cisco (http:/store.theflip.com/en-us/). This device has the capacity to shoot, edit, and share real life experiences in a seamless manner. The authors' experiences with this device include the creation and distribution of video tapings for review; delivery of supplementary educational materials and content; and student assignments of created Flip Video ${ }^{\mathrm{TM}}$.productions, such as with one of the authors' undergraduate nursing students (described later in this review).

Specifications

The four to 16 gigabyte models of the Flip Video record from one to four hours of audio and video material depending on the resolution used to shoot the video (the higher the resolution the more memory required to store). Once recorded, the video is easily uploaded as an MP4 file to a computer via a cable connecting it to a computer. The price ranges from about $\$ 150$ - $\$ 280$ dollars U.S. (at the time of this manuscript) depending on the number of gigabytes. The video quality options are either VGA or High Definition (HD).

Movie production

The Flip Video ${ }^{\mathrm{TM}}$ device has a built-in microphone that simultaneously records audio and visuals by pushing one button. It is handy for travel because of its small size and is as easy to use as a digital camera. One of the authors has used one of these recorders in lieu of higher end video cameras to record high-fidelity patient simulations. There was no loss of fidelity and the device was easy to use for both recording and transferring to a computer for additional editing and storage.

\section{Distribution of video/editing}

The resulting Flip Video files may be transferred to MPG mobile devices (such as Zune and iPod), and/or websites such as YouTube ${ }^{\circledR}$ (2010 YouTube, LLC). In addition they may be posted 
and used in an electronic learning environment or platforms, such as BlackBoard ${ }^{\mathrm{TM}}$ or Moodle. However, transferring video clips to these respective devices may require additional software.

\section{Apple iPhone ${ }^{\mathrm{TM}}$}

The iPhone (currently iPhone 4 with an approaching iPhone 5 release) is one of the most popular Apple products in quite some time. It has popularized touchscreens on a small surface. The iPhone is able to shoot still photos, record video, and play music among other features. In fact, one may actually edit the video, in a basic fashion, to include a song from songs stored on the device.

\section{Specifications}

The iPhone 4 is available in 2 models, 16 or 32 gigabytes (GB) of memory. It weighs slightly less than 5 ounces. The $3 \mathrm{GS}$ (with $8 \mathrm{~GB}$ of memory) is from the last model year and is now less than $\$ 50$ dollars U.S. (including a 2-year contract from AT\&T). In addition to being limited in memory this model has only one camera. Nonetheless, the $3 \mathrm{GS}$ still works well with many, if not most, of the iPhone 4 applications.

\section{Movie production}

The iPhone 4 has 2 cameras (one in the front screen and one in the back of the phone). The camera on the back area of the phone has a 5 megapixel resolution for still photographs and is also able to record video at a decent resolution of $720 \mathrm{p}$ at 30 frames per second with an audio track. A light emitting diode (LED) flash is included for this camera to help with low-light still shots, too. Basic editing for video or still photographs may be performed on the phone (cutting, re-arranging frames, etc.) as well. However more detailed editing is possible using the iMovie or iPhoto applications (Mac-only). More detailed editing also may be done on PC or Mac using a product such as Adobe Photoshop or equivalent. 
The camera facing the user, referred to as the front camera, is intended for videoconferencing with colleagues or friends. It is able to video conference with VGA quality video at 30 frames per second along with audio. The iPhone comes with its own video-conferencing software that is called "FaceTime."

The iPhone and iPod devices use the iTunes center to transfer files between the computer (Apple or PC) and iPhone / iPod. There is also proprietary software, such as 4Media's iPhone .Transfer ${ }^{\mathrm{TM}}$ (http://www.mp4converter.net/iphone-transfer.html), available to assist with moving files between computers and mobile media devices.

\section{Distribution}

Like the Flip Video, after editing is completed the video clips may be distributed to YouTube, included as attachments with texting on some phones, or as an email attachment, in three different sizes (medium, large or high-definition). Medium is $640 \times 480$, large is $960 \times 540$ and high definition is $1280 \times 720$. Keep in mind as the resolution size increases, so does the file size. Also remember the file size will impact how quickly the file uploads. Once the video is distributed to YouTube one may then embed or share the link with other contacts. Additional specifications not mentioned here are clearly available and outlined on the Apple website (htp://www.apple.com/iphone/specs.html), as well as listed in the Table at the end of the paper.

\section{Android}

The Android operating system, released in 2008, is the software basis of a newer generation of smartphones. Google is the company known for developing and growing this operating system along with other members of the Open Handset Alliance (htp//www openhandsetalliance.com/). As with the iPhone, the Android system is a graphical, touch-based way to interact with a Linux-based operating system (OS). 
Because there are many different types of smartphones based on the Android OS, this review will only touch on generalities. A word of warning: Android-run smartphones may have different versions of the OS, which may impact $\mathrm{A} / \mathrm{V}$ editing on the phone.

Specifications

Most, if not all, Android-powered phones are able to record and playback video clips. Many record in high-definition using 5MP to 8MP cameras with 720 pixels video capture resolution and the ability to playback at up to 1080 pixel HD output.

Movie Production

Most Android-based phones do not come with a video editing application. You will need to purchase one from the Android Application store. There are also some free editing applications one may want to use such as VideoPad Video Editor (http:/www.nchsoftware.com/videopad/index.html?gclid=CKmc aXuz6sCFQjCKgodlgi6Sw).

\section{Educational Potential}

There is creative educational potential for the use of video clips, both by students and healthcare professional faculty. Nursing students, for example, may record group assignments, such as an educational session, and deliver the project to the class via a computerized system such as YouTube or electronic learning platforms, such as BlackBoard ${ }^{\mathbf{T M}}$ or Moodle. Or, the videos may be streamed on patient oriented social networks, such as Tudiabetes (http:/www.tudiabetes.org/) or Diabetes University (http://www.diabetes-university.com/). One West Coast University makes the Flip Video available to students from the University's Center for Instructional Technology (CIT). The Flip Videos may be used after minimal instruction on their use for required educational video projects. This provides an opportunity for students to tap 
into their creative ability and learn skills about film production that may be useful in their future professional lives.

After an educational project at the West Coast University, one student enthusiastically told the instructor he was very inspired by designing the creative multimedia course project and wanted to learn medical film production in the future. In using the Flip Video recorder, students have demonstrated innovative planning, filming, editing, and showcasing the projects to classmates for disseminated learning. It has also allowed them to evaluate, and learn from each other's efforts, both in the quality of the video, and the quality of the educational material produced.

Furthermore, with permission of professional conference organizers and speakers, these types of devices may be used to record speakers at conferences for use with colleagues and students who were not able to attend the conference. Always being mindful of both copyright and privacy, creative faculty, clinicians, and students will find many uses for mobile cameras in nursing care and education.

Before making a video that involves patients or students be sure that you are not violating HIPPA or other legalities. When in the planning stages it may be prudent to consult with a health and HIPPA-wise legal source. This is a wise course of action whether taping a conference to share with a colleague, recording a student in their clinical setting for evaluation purposes, or videoing a procedure for a patient to use at home.

\section{Closing thoughts}

The Institute of Medicine (IOM, 2010) recently asked healthcare professional educators to look closely at innovative methods of teaching future nurses. One such method is the use of video recorders that allow learners to demonstrate both learning and imagination. Like any 
technological tool, the tool in and of itself does not have any inherent ingenuity value. However, it is what the user does with the easy to use tool that enhances the individual's learning and assists with meeting certain goals and objectives. Video recording allows healthcare professionals, educators, and students to deliver appropriate learning material that meets educational goals in an original and engaging manner. Plus, these devices are easy to carry and fun to use! As a well known multimedial instructional designer stated: "I shall argue that we should take the tools away from the instructional designers and give them to the learners, as tools for knowledge construction rather than media of conveyance and knowledge acquisition" (Jonassen, 1994). 


\section{References and Sites}

Institute of Medicine (2010). The future of nursing: Leading change, advancing health. Retrieved from http:/www iom.edu/Reports/2010/The-Future-of-Nursing-Leading-Change-Advancing-

Health.aspx, accessed November 10, 2010

Jonassen, D. H. (1994, May 3). Technology as cognitive tools: Learners as designers.

(LISTSERV ITFORUM@UGA.CC.UGA.EDU).

Apple: http:/www.apple.com

Blackbloard: http:/www.blackboard.com

Facebook: http:/www. facebook.com

Flip Video ${ }^{\mathrm{TM}}$ : http://www theflip.com

Moodle: http://moodle.org

Open Handset Alliance: http://www.openhandsetalliance.com/

YouTube (C 2010 YouTube, LLC): http://www.youtube.com

\section{Note:}

Please contact Dr. Margaret Hansen (maggie2hansen@gmail.com) to view an example of a student group video project. 